NOUVELLE

\section{Les lymphocytes T CD8* cytotoxiques aggravent le remodelage cardiaque délétère post-ischémique}

Jérémie Lemariél, ${ }^{1,2}$, Icia Santos-Zas ${ }^{1}$, Hafid Ait-Oufella ${ }^{1,3}$
$>$ La prise en charge de l'infarctus du myocarde a connu des progrès considérables au cours des dernières décennies, grâce à la revascularisation coronaire en urgence et aux traitements antithrombotiques, permettant de réduire la mortalité précoce à moins de $5 \%$ [1]. Ces résultats encourageants ne peuvent cependant occulter le problème croissant des complications à long terme, telles que les arythmies, l'insuffisance cardiaque et les hospitalisations répétées. Les efforts de recherche ciblant les voies pathogéniques impliquées dans le remodelage cardiaque délétère postischémique doivent donc être poursuivis. L'implication de la réponse immunitaire dans les complications cardiaques à long terme après un infarctus du myocarde est largement documentée par des études expérimentales et cliniques. Au décours de l'occlusion artérielle coronaire, l'interruption de l'apport sanguin entraîne la mort rapide des cardiomyocytes, libérant des signaux de danger qui favorisent le recrutement local de cellules inflammatoires. Ces acteurs cellulaires participent à l'élimination des débris apoptotiques, mais peuvent eux-mêmes induire des dommages tissulaires en libérant des cytokines, des protéases et des radicaux libres [2]. Chez la souris, l'infiltration massive du myocarde par des granulocytes neutrophiles dans les 24 premières heures est suivie par celle de deux souspopulations de monocytes: Ly6-Chigh et Ly6-Clow [3]. Les monocytes Ly6-Chigh dominent la phase aiguë pendant les quatre premiers jours et contribuent au remodelage tissulaire défavorable. Les monocytes Ly6-Clow sont recrutés au $4^{e}$ ou $5^{e}$ jour et orchestrent, avec les macrophages cardiaques résidents, la cicatrisation et l'angiogenèse [4]. Des mécanismes similaires ont également été décrits chez l'homme [5]. Des travaux plus récents ont mis en évidence un rôle des lymphocytes $T$ CD $4^{+}$et des lymphocytes $B$ dans le remodelage post-ischémique du myocarde [6-8]. $\varepsilon$ n revanche, le rôle des lymphocytes $T$ cytotoxiques $\operatorname{CD} 8^{+} n^{\prime}$ est pas connu.

\section{Modèle murin d'ischémie cardiaque permanente}

À l'aide d'un modèle d'infarctus du myocarde par ligature de l'artère coronaire chez des souris (57BL/6), nous avons montré que les lymphocytes $T$ $\mathrm{CD} 8^{+}$sont recrutés au sein du myocarde infarci ${ }^{1}$, mais également en bordure de la zone ischémique. La cinétique de recrutement est rapide, dès le premier jour après l'infarctus du myocarde, avec un pic de recrutement au $3^{e}$ jour (Figure 1). Le recrutement des lymphocytes T CD8 ${ }^{+}$est en grande partie orchestré par les lymphocytes $\mathrm{T} \mathrm{CD4}^{+}$. Classiquement, après leur activation à la suite de la reconnaissance d'une cible cellulaire (comme une cellule cancéreuse ou infectée par un virus), les lymphocytes $\mathrm{T}^{\mathrm{C}} \mathrm{C} 8^{+}$entraînent l'apoptose de leur cible par le relargage de granzymes et l'expression du récepteur FAS (Fas cell surface death receptor). Nous avons observé que les

\footnotetext{
Myocarde nécrosé par infarctus.
}

'Université de Paris, Inserm U970, Paris centre de recherche cardiovasculaire, 56 rue Leblanc, 75015 Paris, France.

${ }^{2}$ Service de médecine intensive-réanimation, CHU de Nantes, 44093 Nantes, France.

${ }^{3}$ Service de médecine intensive-réanimation, Hôpital Saint-Antoine, AP-HP, Sorbonne Université, 75012 Paris, France.

hafid.aitoufella@inserm.fr

lymphocytes $T C D 8^{+}$recrutés au sein du myocarde étaient activés, comme l'atteste l'expression membranaire de CD69 et de CD107a, et qu'ils libéraient localement du granzyme B (Figure I), suggérant leur implication dans la réponse immunitaire post-ischémique cardiaque [9].

Afin d'évaluer directement l'implication des lymphocytes $T \mathrm{CD}^{+}$dans le remodelage cardiaque post-ischémique, nous avons diminué le nombre de ces cellules cytotoxiques par l'injection aux souris d'un anticorps monoclonal spécifique anti-CD8 une heure après la ligature de l'artère coronaire. La déplétion en lymphocytes $T C D 8^{+} n^{\prime} a$ pas modifié significativement la mortalité post-infarctus, mais elle a limité le remodelage cardiaque et amélioré les fonctions cardiaques diastolique et systolique (évaluées par échocardiographie et cathétérisme cardiaque). En histologie, la taille de la zone infarcie (Figure 2A) et la fibrose interstitielle (Figure 2B) étaient significativement réduites chez ces souris [9].

En utilisant trois modèles expérimentaux complémentaires, nous avons montré que l'activité pathogène des lymphocytes T CD8 ${ }^{+}$impliquait l'engagement de leur récepteur TCR ( $T$-cell receptor), donc la reconnaissance d'un antigène. Le premier modèle a consisté à produire un infarctus du myocarde chez des souris OT-I, dont les lymphocytes $T \mathrm{CD}^{+}$ne reconnaissent que l'ovalbumine (qui n'est pas exprimée naturellement chez la souris). La déplétion de ces souris en lymphocytes 

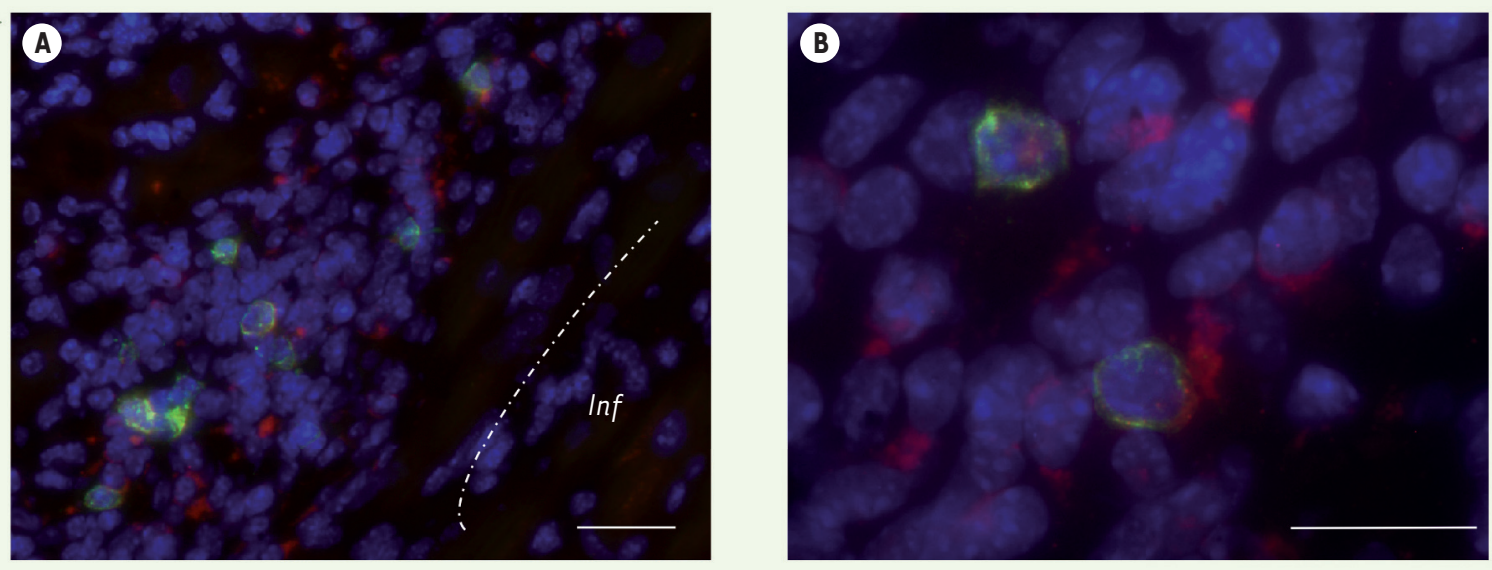

Figure 1. Immunomarquage fluorescent trois jours après un infarctus du myocarde expérimental chez la souris. On voit, autour de la zone infarcie (dont la limite est indiquée par le trait en pointillé), une infiltration du myocarde par des lymphocytes T CD8 $8^{+}$(colorés en vert), qui libèrent du granzyme B (coloré en rouge) au contact des cardiomyocytes. Les noyaux des cellules sont colorés en bleu par le DAPI. Barre d'échelle $=40 \mu \mathrm{m}$. Figure adaptée de [9].

$\mathrm{TCD}^{+}$ne leur conférait aucune protection, concernant notamment la taille de l'infarctus (Figure 1D). Le deuxième modèle a consisté à supplémenter des souris $\mathrm{Ragl}^{-/}$(qui ne possèdent pas de lymphocytes B ou $T$ matures) avec des lymphocytes T CD $8^{+}$«normaux » ou avec des lymphocytes T CD $8^{+}$provenant de souris OT-1. Le transfert de lymphocytes $\mathrm{T} \mathrm{CD}^{+}$de souris OT-I (donc incapables de reconnaître un antigène spécifique par leur TCR) induisait moins de dégâts myocardiques (taille de l'infarctus réduite et meilleure fonction cardiaque) que le transfert de lymphocytes $T \mathrm{CD}^{+}$de souris témoins. Le troisième modèle a tiré profit de la souche de souris transgéniques CMymOva, dont les cardiomyocytes expriment spécifiquement l'ovalbumine et peuvent donc être reconnus par le TCR des lymphocytes T CD8 ${ }^{+}$de souris OT-I. L'injection de lymphocytes $T \mathrm{CD}^{+}$de souris OT-I trois jours avant la réalisation d'une occlusion de l'artère coronaire induisait une augmentation très significative de la mortalité et une majoration de la taille de l'infarctus par rapport à l'injection de lymphocytes T $C D 8^{+}$de souris témoins [9]. L'ensemble de ces résultat suggérait que la stimulation du TCR des lymphocytes $\mathrm{T} C D 8^{+}$participe à leur activité pathogène dans le contexte de l'ischémie cardiaque aiguë.

Afin de mieux comprendre les mécanismes délétères impliquant les lymphocytes $\mathrm{T} \mathrm{CD}^{+}$au cours de l'infarctus du myocarde, nous avons analysé les effets de la déplétion en lymphocytes $\mathrm{T} C D 8^{+}$sur la réponse immunitaire dans le cœur au cours de l'infarctus du myocarde. Par cytométrie de flux et analyse histologique, nous avons montré que la déplétion n'affectait pas le recrutement myocardique des autres sous-populations de cellules myéloïdes et lymphoïdes. La cytotoxicité des lymphocytes T CD $8^{+}$dans le contexte du cancer ou d'une infection virale implique le relargage de perforine/granzyme B [10]. Nous avons mis en évidence que la déplétion de ces lymphocytes entraînait une diminution de l'expression de granzyme $B$ au sein du myocarde ischémique, accompagnée d'une réduction significative de l'apoptose des cellules myocardiques, d'une diminution des taux intra-tissulaires de cytokines pro-inflammatoires et de l'activité des métalloprotéinases impliquées dans la dégradation de la matrice extracellulaire. La constatation des mêmes modifications de la réponse inflammatoire post-ischémique chez des souris déficientes en granzyme B (souris $\mathrm{Gzm}^{-/-}$) a permis de confirmer le rôle cytotoxique direct de cette enzyme. Les expériences de co-culture de lymphocytes $\mathrm{TCD}^{+}$et de cardiomyocytes ont ensuite confirmé ce résultat in vitro. En présence de cellules cardiaques, la culture de lymphocytes $T \mathrm{CD}^{+}$témoins en faible quantité entraînait une altération de la contractilité des cardiomyocytes, et en plus forte quantité, elle induisait leur apoptose. Cet effet cytotoxique n'était plus observé avec des lymphocytes $T$ CD8 ${ }^{+}$déficients en granzyme $B$ [9].

\section{Modèle porcin d'ischémie cardiaque transitoire}

Pour confirmer le rôle pathogène des lymphocytes T CD $8^{+}$dans un modèle expérimental plus pertinent et évaluer le potentiel thérapeutique d'un anticorps anti-CD8, nous avons utilisé un modèle d'ischémie-reperfusion cardiaque chez le porc. Nous avons montré que l'administration d'un anticorps monoclonal anti-CD8 porcin induisait une déplétion en lymphocytes $T$ 


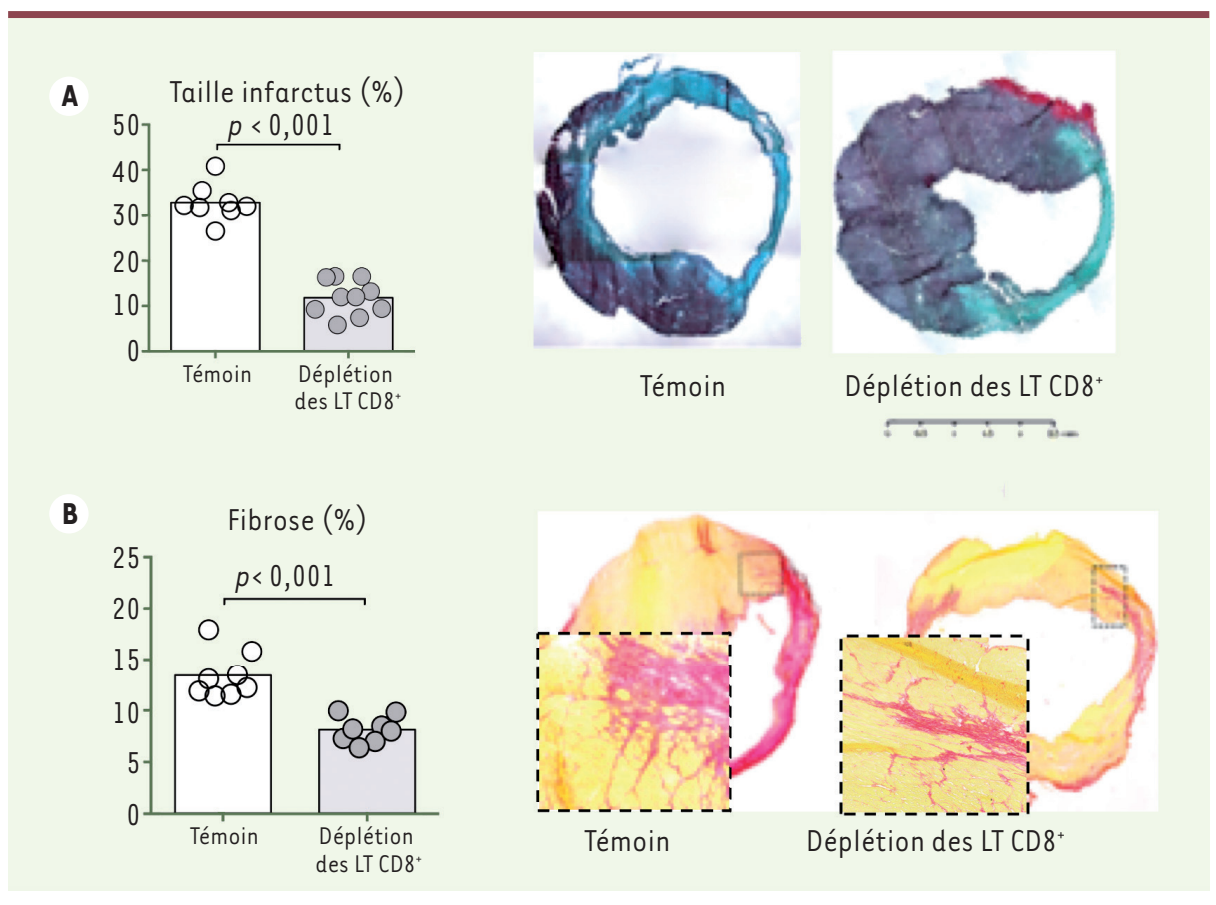

Figure 2. La déplétion en lymphocytes $T C D 8^{*}$ réduit la taille de la zone infarcie (mesurée trois semaines après l'infarctus), ainsi que la fibrose interstitielle. A. Coloration par trichrome de Masson. B. Coloration par rouge Sirius. LT : lymphocytes T. Figure adaptée de [9].

avons réalisé des analyses histologiques sur des biopsies cardiaques humaines, réalisées lors de la pose d'une assistance circulatoire pour un choc cardiogénique compliquant un infarctus du myocarde. Chez ces patients, nous avons détecté l'infiltration du myocarde par des lymphocytes T $\operatorname{CD}^{+}$dès le $3^{e}$ jour après l'infarctus. Les

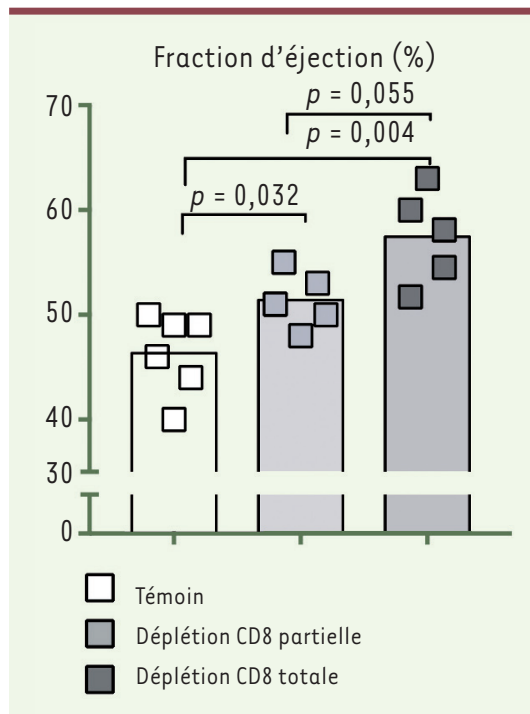

Figure 3. La déplétion en lymphocytes $T C D 8^{+}$ préserve la fonction contractile du ventricule gauche dans un modèle d'infarctus du myocarde reperfusé chez le porc. Analyse échocardiographique deux semaines après l'infarctus. L'effet protecteur est plus important dans le groupe où la déplétion est complète. Figure adaptée de [9].

$\mathrm{CD}^{+}$complète, mais plus lente que chez la souris (trois jours, au lieu de six heures). Nous avons donc adapté le protocole expérimental afin d'ob- tenir, lors de l'ischémie-reperfusion cardiaque, soit une déplétion complète (injection d'anticorps trois jours avant l'occlusion de l'artère coronaire), soit une déplétion partielle (injection une heure après l'occlusion de l'artère coronaire). Les taux plasmatiques de granzyme $B$ étaient effondrés après traitement par l'anticorps anti-CD8. Deux semaines après l'occlusion de l'artère coronaire, la taille de l'infarctus était diminuée de $60 \%$ dans le groupe avec déplétion complète par rapport au groupe témoin $(p<0,01)$. La fonction systolique ventriculaire gauche (évaluée par échocardiographie) était moins altérée chez les porcs traités par l'anticorps anti-CD8, que la déplétion soit complète ou incomplète, mais les bénéfices étaient plus marqués en cas de déplétion complète (Figure 3) [9]. Ces résultats confirment le rôle pathogène des lymphocytes T CD $8^{+}$ dans un modèle porcin d'infarctus du myocarde reperfusé.

\section{Données chez l'homme}

Afin d'évaluer la pertinence clinique de ces résultats expérimentaux, nous lymphocytes T CD8 ${ }^{+}$exprimant le granzyme $B$ étaient principalement détectés dans la zone infarcie au cours de la première semaine, puis dans la zone bordant la zone infarcie après le $7^{e}$ jour. Enfin, nous avons mesuré le taux plasmatique de granzyme $B$ dans la cohorte française FAST-MI [1], qui regroupe 1046 patients hospitalisés pour un syndrome coronarien aigu pris en charge dans les unités de soins intensifs cardiologiques en France, et suivis sur une période d'au moins 12 mois. Nous avons alors constaté que les patients ayant un taux de granzyme $B$ élevé (> 8,9 pg/mL) lors de leur admission dans l'unité de soins intensifs présentaient un risque de décès à un an plus important, et ce après ajustement prenant en compte de nombreux autres facteurs de risque (hazard ratio 2,3 $[1,2-4,2] ; p<0,01)[9]$.

\section{Perspective}

Nous avons donc identifié le rôle pathogène des lymphocytes $T \mathrm{CD}^{+}$au cours du remodelage cardiaque post-ischémique. Le mécanisme moléculaire principal repose sur l'effet cytotoxique du granzyme B, qui aggrave la mort des cardiomyocytes et augmente la réponse 
inflammatoire locale [9]. Les résultats de cette recherche physiopathologique ouvrent une nouvelle perspective thérapeutique pour lutter contre l'insuffisance cardiaque consécutive à un infarctus du myocarde : la déplétion en lymphocytes $\mathrm{T} \mathrm{CD}^{+}$en phase aiguë de l'infarctus. $\diamond$

Cytotoxic CD8* T cells promote granzyme B-dependent adverse post-

ischemic cardiac remodeling

\section{LIENS D'INTÉRÊT}

Les auteurs déclarent n'avoir aucun lien d'intérêt concernant les données publiées dans cet article.

\section{RÉFÉRENCES}

1. Puymirat $\varepsilon$, Simon T, Steg PG, et al. Association of changes in clinical characteristics and management with improvement in survival among patients with ST-elevation myocardial infarction. JAMA $2012 ; 308$ : 998-1006.

2. Frangogiannis NG. The inflammatory response in myocardial injury, repair and remodeling. Nat Rev Cardiol $2014 ; 11: 255-65$.

3. Nahrendorf M, Swirski FK, Aikawa $\varepsilon$, et al. The healing myocardium sequentially mobilizes two monocyte subsets with divergent and complementary functions. J Exp Med 2007 ; 204 : 3037-47.

4. Heidt T, Courties G, Dutta P, et al. Differential contribution of monocytes to heart macrophages in steady-state and after myocardial infarction. Circ Res 2014 ; 115 : 284-95.

5. Peet C, Ivetic A, Bromage DI, et al. Cardiac monocytes and macrophages after myocardial infarction. Cardiovasc Res 2020 ; 116 : 1101-12.
6. Zouggari Y, Ait-Oufella H, Bonnin P, et al. B lymphocytes trigger monocyte mobilization and impair heart function after acute myocardial infarction. Nat Med 2013; $19: 1273-80$.

7. Hofmann U, Frantz S. Role of lymphocytes in myocardial injury, healing, and remodeling after myocardial infarction. Circ Res 2015 ; 116 : 354-67.

8. Santos-Zas I, Lemarie J, Tedgui A, Ait-Oufella H. Adaptive immune responses contribute to postischemic cardiac remodeling. Front Cardiovasc Med $2018 ; 5: 198$

9. Santos-Zas I, Lemarié J, Zlatanova I, et al. Cytotoxic $\mathrm{CD}^{+} \mathrm{T}$ cells promote granzyme $\mathrm{B}$-dependent adverse post-ischemic cardiac remodeling. Nat Commun $2021 ; 12: 1483$.

10. Voskoboinik I, Whisstock JC, Trapani JA. Perforin and granzymes: function, dysfunction and human pathology. Nat Rev Immunol 2015 ; 15 : 388-400. 